\title{
International recommendations on the diagnosis and treatment of acquired hemophilia A
}

\author{
Andreas Tiede, ${ }^{1}$ Peter Collins, ${ }^{2}$ Paul Knoebl, ${ }^{3}$ Jerome Teitel, ${ }^{4}$ Craig Kessler, ${ }^{5}$ Midori \\ Shima, ${ }^{6}$ Giovanni Di Minno, ${ }^{7}$ Roseline d'Oiron, ${ }^{8}$ Peter Salaj, ${ }^{9}$ Victor Jiménez- \\ Yuste, ${ }^{10}$ Angela Huth-Kühne ${ }^{11}$ and Paul Giangrande ${ }^{12}$ \\ ${ }^{1}$ Hannover Medical School, Department of Hematology, Hemostasis, Oncology and Stem \\ Cell Transplantation, Hannover, Germany; ${ }^{2}$ Arthur Bloom Haemophilia Centre, University \\ Hospital of Wales School of Medicine, Cardiff University, Cardiff, UK; ${ }^{3}$ Department of \\ Medicine 1, Division of Hematology and Hemostasis, Medical University of Vienna, Vienna, \\ Austria; ${ }^{4}$ Division of Hematology and Oncology, St. Michael's Hospital, Toronto, and \\ Department of Medicine, University of Toronto, Toronto, Canada; ${ }^{5} \mathrm{Georgetown}$ University \\ Hospital, Lombardi Cancer Center, Division of Hematology/Oncology, Washington, DC, USA; \\ ${ }^{6}$ Department of Pediatrics, Nara Medical University, Nara, Japan; ${ }^{7}$ Regional Reference \\ Center for Coagulation Disorders, Federico II University Hospital, Naples, Italy; ${ }^{8}$ Centre de \\ Référence de l'Hémophilie et des Maladies Hémorragiques Constitutionnelles Rares, \\ Hôpitaux Universitaires Paris Sud, Hôpital Bicêtre APHP, Le Kremlin-Bicêtre, France; \\ Institute of Hematology and Blood Transfusion, Prague, Czech Republic; ${ }^{10} \mathrm{Hematology}$ \\ Department, La Paz University Hospital, Autonoma University, Madrid, Spain; ${ }^{11} \mathrm{SRH}$ \\ Kurpfalzkrankenhaus Heidelberg GmbH and Hemophilia Center, Heidelberg, Germany and \\ ${ }^{12}$ Green Templeton College, University of Oxford, Oxford, UK
}

\section{ABSTRACT}

\begin{abstract}
A cquired hemophilia A (AHA), a rare bleeding disorder caused by neutralizing autoantibodies against coagulation factor VIII (FVIII), occurs in both men and women without a previous history of bleeding. Patients typically present with an isolated prolonged activated partial thromboplastin time due to FVIII deficiency. Neutralizing antibodies (inhibitors) are detected using the Nijmegen-modified Bethesda assay. Approximately $10 \%$ of patients do not present with bleeding and, therefore, a prolonged activated partial thromboplastin time should never be ignored prior to invasive procedures. Control of acute bleeding and prevention of injuries that may provoke bleeding are top priorities in patients with AHA. We recommend treatment with bypassing agents, including recombinant activated factor VII, activated prothrombin complex concentrate, or recombinant porcine FVIII in bleeding patients. Autoantibody eradication can be achieved with immunosuppressive therapy, including corticosteroids, cyclophosphamide and rituximab, or combinations thereof. The median time to remission is 5 weeks, with considerable interindividual variation. FVIII activity at presentation, inhibitor titer and autoantibody isotype are prognostic markers for remission and survival. Comparative clinical studies to support treatment recommendations for AHA do not exist; therefore, we provide practical consensus guidance based on recent registry findings and the authors' clinical experience in treating patients with AHA.
\end{abstract}

\section{Introduction}

Acquired hemophilia A (AHA) is characterized by neutralizing autoantibodies, called inhibitors, against factor VIII (FVIII). ${ }^{1}$ AHA is a rare disorder, affecting men and women of all ages. ${ }^{2}$ Two peaks in AHA incidence are typically observed; one associated with pregnancy, and another with older age ( $>60$ years old). Approximately half of patients with AHA have concomitant disorders, most often other autoimmune disorders or malignancy., In approximately $1-5 \%$ of cases, AHA is diagnosed during pregnancy or within 1 year following childbirth. ${ }^{5}$ The bleeding phenotype of AHA is variable, ranging from life-threatening bleeds to

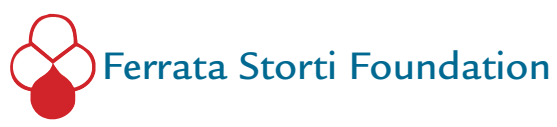

Haematologica 2020

Volume 105(7):1791-1801

\section{Correspondence:}

ANDREAS TIEDE

tiede.andreas@mh-hannover.de

Received: June 27, 2019.

Accepted: April 7, 2020.

Pre-published: May 7, 2020.

doi:10.3324/haematol.2019.230771

Check the online version for the most updated information on this article, online supplements, and information on authorship \& disclosures: www.haematologica.org/content/105/7/1791

(C)2020 Ferrata Storti Foundation

Material published in Haematologica is covered by copyright. All rights are reserved to the Ferrata Storti Foundation. Use of published material is allowed under the following terms and conditions:

https://creativecommons.org/licenses/by-nc/4.0/legalcode. Copies of published material are allowed for personal or internal use. Sharing published material for non-commercial purposes is subject to the following conditions:

https://creativecommons. org//icenses/by-nc/4.0/legalcode, sect. 3. Reproducing and sharing published material for commercial purposes is not allowed without permission in writing from the publisher. 
mild or no bleeding. Subcutaneous hematomas are characteristic of AHA and can be the first indication of the disease.

Patients with AHA are often elderly; comorbidities and medications, such as antiplatelet agents and anticoagulants, may influence the clinical picture and require an individualized therapeutic approach. In contrast to congenital hemophilia, comparative clinical studies are not available in AHA, largely because of the rarity of the disorder and the severe clinical condition of patients at presentation. Treatment decisions are often based on the expertise and clinical experience of treating physicians, and referral to expert centers is often recommended to provide the best possible care.

In 2009, Huth-Kühne et al. published international recommendations for AHA. ${ }^{1}$ Since then, other guidance has also been published. ${ }^{6}$ These documents are recognized as important sources of guidance for hematologists and other specialists. The 2009 recommendations were mainly based on the authors' collective experience in treating a large number of patients with AHA. Data from several AHA registries have since been published, including the EACH2 (European ACquired Haemophilia), 3,5,7,8 SACHA (Surveillance des Auto-antiCorps au cours de l'Hémophilie Acquise) ${ }^{9}$ and GTH (Gesellschaft für Thrombose- und Hämostaseforschung) registries ${ }^{10-13}$ in Europe, as well as the HTRS (Hemostasis and Thrombosis Research Society) registry in the USA. ${ }^{14}$ Moreover, a clinical trial investigating the use of a recently introduced treatment for AHA, recombinant porcine FVIII (rpFVIII), has been reported..$^{15}$ Here, we provide an updated set of recommendations based on this higher level of recent evidence, which has influenced clinical practices in AHA.

\section{Methods}

First, each author independently reviewed the 2009 international AHA recommendations, ${ }^{1}$ identifying areas in which an update was required based on their personal experience and knowledge of current literature. Feedback was consolidated in a single document, and the latest available published evidence was assessed to ascertain the extent to which each proposed statement was justified, with particular emphasis on the results of the AHA registries summarized in Table 1. A PubMed literature search was conducted to identify additional relevant publications published since 2009. The search strategy and a PRISMA diagram are provided in the Online Supplementary Material.

Recommendations were formulated according to Guyatt et al., ${ }^{16}$ as detailed in Online Supplementary Table S1. We used GRADE 1 for recommendations ("we recommend") whenever a clear impact on patients' safety or benefit would outweigh risks and burden. More specifically, GRADE $1 \mathrm{~B}$ was used when the statement was supported by data from at least one observational or interventional study, and when the recommendation seemed to apply to most patients in most circumstances without reservation; GRADE 1C was used for recommendations that lacked support from such evidence, but still appeared to be important for patients' safety or benefit. GRADE 2 ("we suggest") was used for weaker suggestions (2B for those with support from registries or studies, and 2C without) that may change when new data become available. Table 2 lists our main recommendations in the order in which they are discussed.

\section{Diagnosis}

AHA is rare, usually occurring unexpectedly, with physicians of different specialties potentially seeing patients initially. Therefore, a simplified diagnostic algorithm to assist physicians who may not have direct experience of AHA is required (Figure 1).

Typically, patients with AHA present with acute or recent bleeding symptoms, without a previous history of bleeding, with laboratory investigations showing an isolated prolonged activated partial thromboplastin time (APTT), reduced FVIII activity (FVIII:C) $(<1 \%$ in $50 \%$ of cases; $<5 \%$

Table 1. Recent studies and registries in acquired hemophilia A.

\begin{tabular}{|c|c|c|c|c|c|c|c|c|c|c|}
\hline \multirow[t]{2}{*}{ Study name } & \multirow[t]{2}{*}{$\begin{array}{l}\text { Study } \\
\text { type }\end{array}$} & \multirow[t]{2}{*}{ Design } & \multirow[t]{2}{*}{$\begin{array}{l}\text { Collection } \\
\text { period }\end{array}$} & \multirow[t]{2}{*}{$\begin{array}{l}\text { Total } n . \\
\text { of patients }\end{array}$} & \multicolumn{3}{|c|}{$\begin{array}{l}\text { Treatment/outcome data } \\
\text { available information }\end{array}$} & \multirow[b]{2}{*}{$\begin{array}{l}\text { Remission } \\
\text { (n. of pts) }\end{array}$} & \multirow{2}{*}{$\begin{array}{c}\text { Survival } \\
\text { information } \\
\text { (pts) }\end{array}$} & \multirow[t]{2}{*}{ Reference } \\
\hline & & & & & $\begin{array}{c}\text { Hemostatic } \\
\text { therapy } \\
\text { (n. of pts)* }\end{array}$ & $\begin{array}{l}\text { Bleeding } \\
\text { resolved } \\
\text { (n. of pts or } \\
\text { episodes) }\end{array}$ & $\begin{array}{c}\text { IST } \\
\text { (n. of pts) })^{* *}\end{array}$ & & & \\
\hline $\begin{array}{l}\text { UK surveillance } \\
\text { study }\end{array}$ & Registry & $\begin{array}{l}\text { Prospective, } \\
\text { consecutive }\end{array}$ & 2001-2003 & 172 & 97 & - & 151 & 105 & 113 & (2) \\
\hline EACH2 & Registry & $\begin{array}{l}\text { Retrospective } \\
\text { (3 years); } \\
\text { prospective } \\
\text { (3 years) }\end{array}$ & 2003-2009 & 501 & 307 & $\begin{array}{c}288 \text { patients } \\
\text { (1st episodes) }\end{array}$ & 331 & 331 & 331 & $(3,5,7,8)$ \\
\hline SACHA & Registry & Prospective & 2001-2006 & 82 & 38 & 38 patients & 77 & 77 & 82 & (9) \\
\hline GTH-AH 01/2010 & Registry & Prospective & 2010-2013 & 102 & 70 & 162 episodes & 101 & 101 & 102 & $(10-12,37)$ \\
\hline HTRS & Registry & Prospective & 2004-2011 & 166 & 68 (rFVIIa only) & 139 episodes & - & - & & (14) \\
\hline OBI-1 & Clinical trial & $\begin{array}{l}\text { Prospective, } \\
\text { single-arm }\end{array}$ & - & 29 & 28 (rpFVIII only) & 28 patients & - & - & 29 & (15) \\
\hline
\end{tabular}


in $75 \%$ of cases; $<40 \%$ in $100 \%$ of cases), and the presence of autoantibodies, detected by the Bethesda assay or by enzyme-linked immunosorbent assay (ELISA). ${ }^{10}$ If the prothrombin time (PT) is prolonged, it must be attributed to other reasons, e.g., anticoagulant treatment. The bleeding pattern in AHA is characteristic of the disease, with subcutaneous bleeds being most common (observed in $80 \%$ of patients), followed by muscle, gastrointestinal, genitourinary, and retroperitoneal bleeds (in 45\%, 21\%, 9\% and 9\% of patients, respectively). ${ }^{2}$ Joint bleeds, the hallmark of congenital hemophilia, are much less common in AHA. ${ }^{2,8}$ In some cases, patients with AHA have not yet started to bleed at the time of diagnosis. ${ }^{2}$ In these patients, a prolonged APTT may be the only sign of AHA.

Table 2. Summary of recommendations on the diagnosis and treatment of patients with acquired hemophilia A.

\section{Diagnosis}

- We recommend that the diagnosis of AHA should be considered whenever an acute or recent onset of bleeding is accompanied

- We recommend that an unexplained APTT prolongation prior to surgery should be investigated and not ignored.

- We recommend confirming a diagnosis of AHA by testing FVIII activity and inhibitor concentration using the Bethesda assay and/or an anti-FVIII ELISA

- We recommend testing for anti-porcine inhibitors using a modified Bethesda assay, if treatment with rpFVIII is an option.

\section{Hemostatic treatment}

- We recommend that hemostatic treatment be initiated in patients with AHA and clinically relevant bleeding irrespective of inhibitor titer and residual FVIII activity.

- We recommend the use of rFVIIa, APCC or rpFVIII instead of human FVIII concentrates or desmopressin for the treatment of clinically relevant bleeding in patients with AHA.

- We recommend that alternative treatment strategies from among the first-line agents be used if appropriate initial treatment fails.

- For initial treatment with rFVlla, we recommend bolus injection of $90 \mu \mathrm{g} / \mathrm{kg}$ every $2-3 \mathrm{~h}$ until hemostasis is achieved.

- For initial treatment with APCC, we recommend bolus injections of between 50-100 U/kg every 8-12 h, up to a maximum of $200 \mathrm{U} / \mathrm{kg} / \mathrm{day}$.

- For initial treatment with rpFVIII, we recommend the approved dose of $200 \mathrm{U} / \mathrm{kg}$, followed by further doses to maintain trough levels $>50 \%$.

- We recommend close monitoring of FVIII activity during therapy with rpFVIII.

- We suggest the use of recombinant or plasma-derived human FVIII concentrates only if bypassing agents or rpFVIII are unavailable or ineffective and the inhibitor titer is low. We recommend against the use of desmopressin.

-We recommend the prophylactic use of bypassing agents or rpFVIII to cover minor or major invasive procedures.

\section{Inhibitor eradication}

- We recommend IST in all patients with AHA. However, particular caution should be exercised in frail patients.

- We suggest using prognostic markers (FVIII activity, inhibitor titer, if available) to individualize IST.

- We suggest that patients with FVIII $\geq 1 \mathrm{IU} / \mathrm{dL}$ and inhibitor titer $\leq 20 \mathrm{BU}$ at baseline receive first-line treatment with corticosteroids alone for 3-4 weeks.

- We suggest combining corticosteroids with rituximab or a cytotoxic agent for first-line therapy in patients with FVIII $<1 \mathrm{IU} / \mathrm{dL}$ or inhibitor titer $>20$ BU.

- We suggest extending observation in patients who do not achieve remission with first-line IST but have continued improvement of FVIII activity and inhibitor titer.

- We suggest second-line therapy with rituximab or a cytotoxic agent, whichever was not used during first-line therapy.

- For corticosteroid therapy, we suggest prednisolone or prednisone at a dose of $1 \mathrm{mg} / \mathrm{kg} / \mathrm{day}$ PO for a maximum of 4-6 weeks (followed by a tapered withdrawal).

- We suggest rituximab at a dose of $375 \mathrm{mg} / \mathrm{m}^{2}$ weekly for a maximum of 4 cycles.

- As cytotoxic therapy, we suggest cyclophosphamide at a dose of $1.5-2 \mathrm{mg} / \mathrm{kg} /$ day PO for a maximum of 6 weeks, or MMF at a dose of $1 \mathrm{~g} /$ day for 1 week, followed by $2 \mathrm{~g} /$ day.

- We do not recommend the use of high-dose human FVIII for immune tolerance induction in AHA.

- We do not recommend the use of high-dose intravenous immunoglobulins for inhibitor eradication in patients with AHA.

- We recommend follow-up after complete remission, using FVIII:C monitoring monthly during the first 6 months, every 2-3 months up to 12 months, and every 6 months during the second year and beyond, if possible.

- In women with pregnancy-associated AHA, we suggest the same approach for IST as in other patients, but with a more careful consideration regarding the use of cytotoxic agents.

- We recommend thromboprophylaxis according to ASH guidelines if FVIII:C has returned to normal levels. If indicated, therapy with anti-platelet drugs or oral anticoagulants should be initiated after normal FVIII:C levels have been achieved.

AHA: acquired hemophilia A; APTT: activated partial thromboplastin time; FVIII: factor VIII; ELISA: enzyme-linked immunosorbent assay; rpFVIII: recombinant porcine FVIII; rFVIIa: recombinant activated factorVII; APCC: activated prothrombin complex concentrate; IST: immunosuppressive therapy; BU: Bethesda unit; PO: orally; MMF: mycophenolate mofetil; IVIG: intravenous immunoglobulins; FVIII:C: factor VIII activity; ASH: American Society of Hematology. 


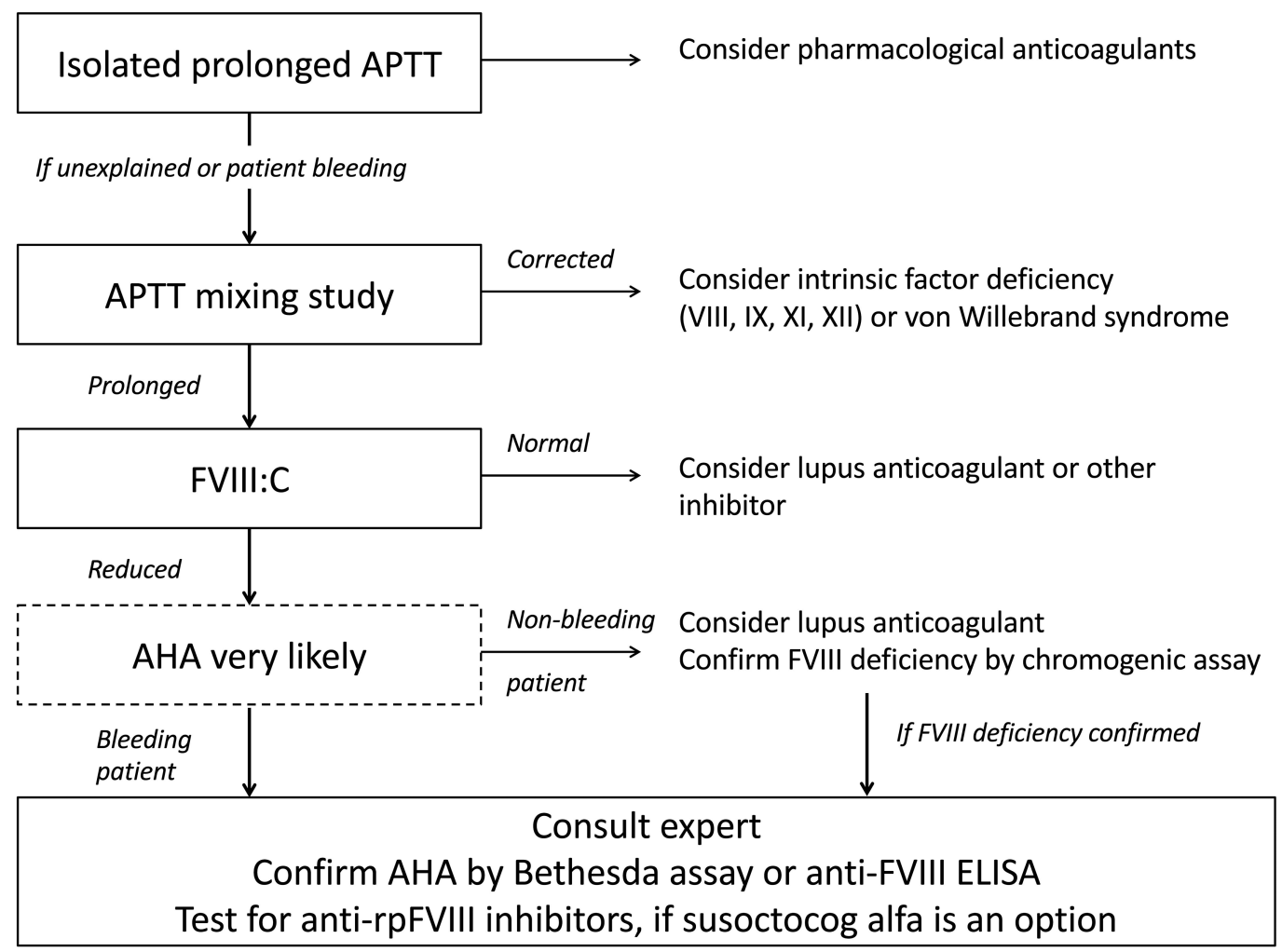

Figure 1. Diagnostic pathway for acquired hemophilia A. The activated partial thromboplastin time (APTT) mixing study will not be needed in an environment in which factor VIII (FVIII) activity is immediately available. Note that the presence of lupus anticoagulant does not exclude acquired hemophilia A. See the 'Diagnosis' section for more details. FVIII:C: factor VIII activity; AHA: acquired hemophilia A; ELISA: enzyme-linked immunosorbent assay; rpFVIII: recombinant porcine factor VIII.

A prolonged APTT is not specific to FVIII deficiency; other causes of APTT prolongation are much more common. Therefore, a prolonged APTT does not constitute a good predictive biomarker for bleeding, as evidenced by UK guidelines recommending against routine testing in unselected patients. ${ }^{17}$ However, we recommend that an unexplained APTT prolongation should not be ignored if it is encountered before surgery or in bleeding patients. Measuring normal FVIII, factor IX (FIX) or factor XI (FXI) levels will exclude a bleeding diathesis in such cases and testing for conditions that prolong the APTT but do not pose a bleeding risk, such as lupus anticoagulant (LA) and factor XII deficiency, may be performed.

We recommend that the diagnosis of AHA should be considered whenever an acute or recent onset of bleeding is accompanied by an unexplained prolonged APTT (GRADE 1B).

We recommend that unexplained $A P T T$ prolongation prior to surgery should be investigated and not ignored (GRADE 1C).

\section{Mixing tests}

Coagulation factor deficiencies or coagulation factor inhibitors, including autoantibodies, LA, or pharmacological anticoagulants, may result in a prolonged APTT. To distinguish a factor deficiency from the presence of an inhibitory substance, mixing tests may be conducted if FVIII:C is not immediately available. AHA FVIII inhibitors are time- and temperature-dependent, so APTT results obtained immediately following the mixture of normal and patient plasma and after a $2 \mathrm{~h}$ incubation should be compared. ${ }^{18}$ These tests are poorly standardized and can- not be used to establish or exclude AHA. ${ }^{19}$ Further investigation is always required, and specific factor activity assays should be performed in parallel to facilitate early diagnosis.

\section{Coagulation factor measurements}

An isolated low FVIII level suggests a diagnosis of AHA. However, differential diagnoses of low FVIII:C include von Willebrand disease, congenital hemophilia $A$ and the acquired von Willebrand syndrome. ${ }^{19}$ A decrease in all intrinsic coagulation factors may be an in vitro false result arising from inhibitor-induced FVIII depletion in the substrate plasma. ${ }^{20}$ LA-induced inhibition of phospholipid in the factor activity assay can also result in reduced factor levels. LA can be excluded by a negative diluted Russell viper venom test, which is typically not affected by FVIII inhibitors. ${ }^{21}$ Vice versa, interference of LA on FVIII activity and the Bethesda assay can be excluded by using chromogenic substrate assays that are usually insensitive to LA. ${ }^{22,23}$ Alternatively, a normal chromogenic assay FVIII:C excludes AHA in cases in which LA decreases one-stage FVIII assay results. However, it should be noted that AHA and LA are both autoimmune disorders that can co-exist in the same patient. ${ }^{24,25}$

\section{Bethesda assay and modifications}

The Bethesda assay was developed to detect and quantify FVIII alloantibodies in congenital hemophilia A that display linear type 1 kinetics. ${ }^{26}$ It is also useful in detecting FVIII inhibitors in AHA, but these often display complex 
and non-linear type 2 kinetics and so the assay may not be able to estimate the true potency of the autoantibody. ${ }^{27}$ By consensus, the dilution closest to a $50 \%$ inhibition of FVIII in normal plasma is selected to estimate the inhibitor titer: ${ }^{28} 1$ Bethesda unit $(\mathrm{BU})=$ the amount of inhibitor that neutralizes $50 \%$ of FVIII:C in normal plasma after incubation for $2 \mathrm{~h}$ at $37^{\circ} \mathrm{C}$. Sometimes several dilutions are close to $50 \%$, introducing some uncertainty in assigning the inhibitor titer. LA may interfere with the Bethesda assay and cause low-positive results. Immunoassays to detect anti-FVIII antibodies may help to distinguish LA from FVIII inhibitors in such cases. ${ }^{13,29}$ The Nijmegen modification has been developed to improve specificity in the detection of low-titer inhibitors. ${ }^{30}$ Pre-analytical heat inactivation of test plasma may improve assay accuracy/sensitivity. ${ }^{31,32}$

\section{Anti-porcine inhibitor titer}

The porcine FVIII inhibitor titer should be quantified if rpFVIII is considered as a treatment option. The assay is performed in the same way as the Bethesda assay, but with rpFVIII as the substrate instead of normal human plasma. In the rpFVIII OBI-1 pivotal clinical trial, patients with an anti-porcine titer of $>20$ BU were excluded. ${ }^{15}$ Lower, albeit detectable, anti-porcine titers had implications for dosing of rpFVIII (see the 'Recombinant porcine FVIII' section for more details).

\section{Anti-factor VIII enzyme-linked immunosorbent assay}

A commercial anti-FVIII ELISA was shown to be sensitive and specific for diagnosing AHA. ${ }^{13}$ It may be particularly helpful when interference with LA is suspected in a positive Bethesda assay, or if the Bethesda assay cannot be performed because rpFVIII has already been given. In addition, determining the anti-FVIII isotype may have prognostic implications, as shown for anti-FVIII IgA. ${ }^{11}$

We recommend confirming a diagnosis of AHA by testing FVIII activity and inhibitor concentration using the Bethesda assay and/or an anti-FVIII ELISA (GRADE 1B).

We recommend testing for anti-porcine inhibitors using a modified Bethesda assay, if treatment with rpFVIII is an option (GRADE 1B).

\section{Hemostatic treatment}

Treatment of bleeds in patients with AHA should be carried out in a specialist center; if immediate referral is not possible, expert advice should be sought. The first priority is to control acute bleeds and to prevent injury, including iatrogenic, that may provoke further bleeding. Surgical interventions and other invasive procedures should be performed only at specialist centers, or with expert advice. If a central venous line is required, it may be preferable to use the femoral vein. Venipuncture should be performed by experienced staff, and blood pressure measured only as often as deemed clinically relevant. Fasciotomy for intramuscular bleeds should be avoided because this can result in uncontrolled bleeding. ${ }^{33,34}$ Early hemostatic therapy may prevent compartment syndrome in most patients or even reverse symptoms of developing compartment syndrome. ${ }^{35}$ In the EACH2 registry, the only parameter that differed between patients who responded to treatment and those who did not was a delay in time to treatment. ${ }^{8}$

The decision to initiate treatment is guided by the clini- cal relevance of acute bleeds. Overall, approximately $70 \%$ of patients with AHA need hemostatic treatment. According to data from the UK and the EACH2 registry, approximately $30 \%$ of patients were untreated, ${ }^{2,8}$ apparently because they had no bleeds or only non-severe subcutaneous bleeds. However, close observation may be warranted because even fatal bleeding can occur up to 5 months after first presentation in patients with persistent inhibitors. ${ }^{2}$

A lack of correlation between FVIII:C or inhibitor titer and bleeding phenotype in AHA has been described in many studies. ${ }^{2,3,36}$ Inhibitor titer and FVIII:C should not therefore influence the decision to initiate treatment for clinically relevant bleeding.

Any hemostatic treatment is associated with a risk of arterial and thrombotic events, particularly in elderly patients and those with risk factors or recent thromboembolic events. Acute illness and immobility in bleeding patients with AHA is a risk factor for thromboembolism; however, controlling acute bleeds should usually be prioritized.

We recommend that hemostatic treatment be initiated in patients with AHA and clinically relevant bleeding irrespective of inhibitor titer and residual FVIII activity (GRADE 1B).

\section{Choice of first- and second-line treatment for acute bleeds}

Parallel-group, comparative-treatment studies are not available in AHA. Propensity score-matched analysis of registry data on bypassing agents, including recombinant activated factor VII (rFVIIa; NovoSeven ${ }^{\circledR}$ ) and activated prothrombin concentrate complex (APCC; FEIBA ${ }^{\circledR}$ ), as well as a single-arm clinical trial on rpFVIII did not show a clear efficacy or safety benefit of one drug over the others. ${ }^{8,15,37}$ A similar analysis showed that the efficacy of human FVIII concentrates and desmopressin was clearly lower than that of bypassing agents in EACH2. Therefore, APCC, rFVIIa and rpFVIII can all be considered appropriate first-line treatments (Figure 2). The final choice will be influenced mostly by the anti-porcine titer, as well as availability, cost and ability to monitor rpFVIII.

We recommend the use of rFVIIa, APCC or rpFVIII instead of human FVIII concentrates or desmopressin for the treatment of clinically relevant bleeding in patients with AHA (GRADE 1B).

Patients should be closely monitored for treatment efficacy. Such monitoring is based mainly on clinical judgment. Depending on the bleeding site, serial blood count measurements, inspection and palpation of bleeding sites, patient-reported changes in pain or mobility, as well as imaging studies should be taken into consideration. ${ }^{38}$ When using rpFVIII treatment, monitoring of FVIII:C can also help to guide subsequent dosing, although clinical efficacy may not always correlate with FVIII:C. ${ }^{15}$ Depending on the severity of the condition, failure to improve bleeding symptoms or the appearance of new symptoms may indicate the need for treatment intensification or switching to one of the alternative therapies.

We recommend that alternative treatment strategies from among the first-line agents be used if appropriate initial treatment fails (GRADE 1C).

\section{Recombinant activated factor VII}

The efficacy of rFVIIa [eptacog alfa (activated)] in AHA was recently addressed in a systematic literature review. ${ }^{37}$ A total of 12 studies, reporting on 671 patients and 1,063 


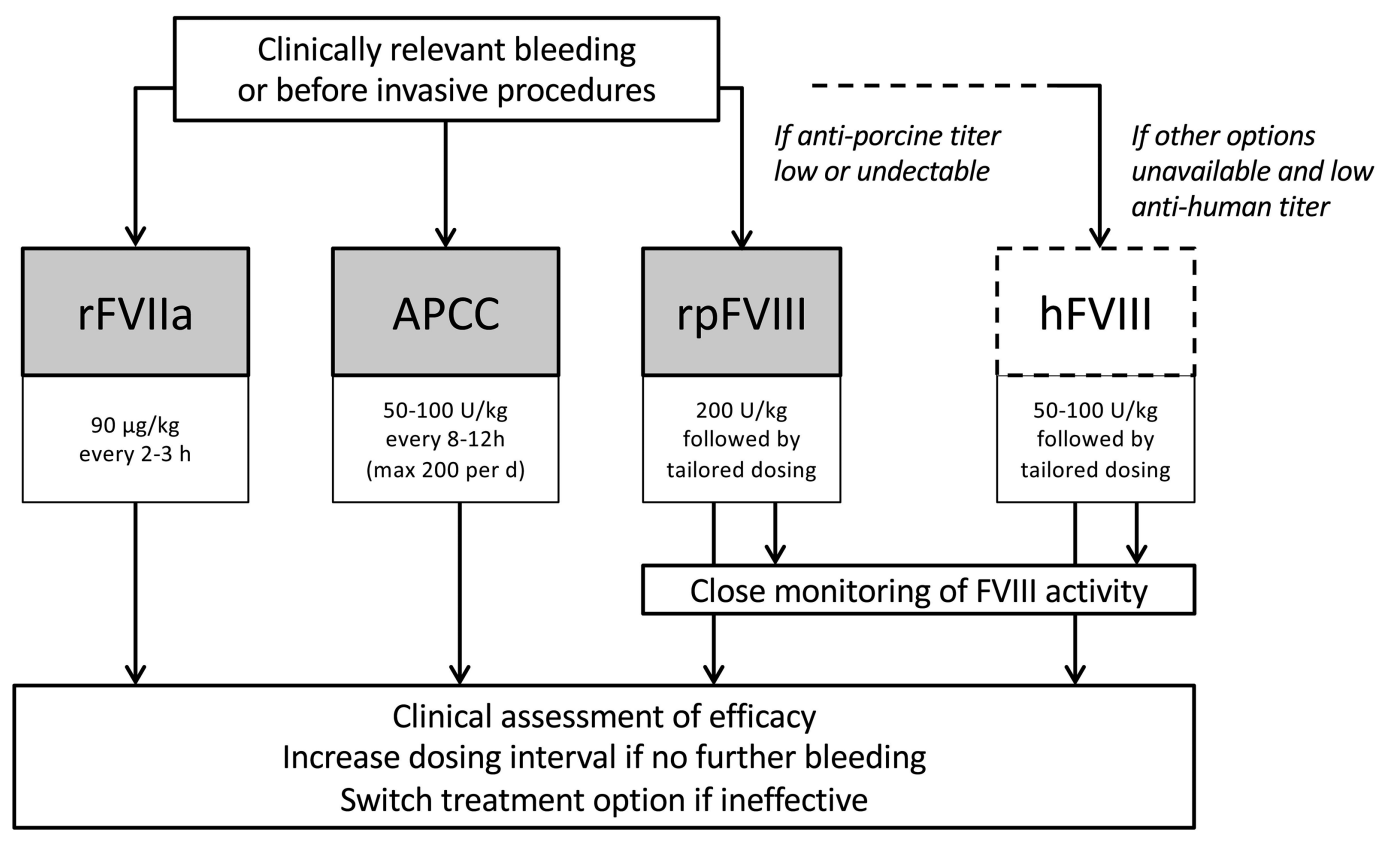

Figure 2. Choice and monitoring of hemostatic therapy in acquired hemophilia A. rFVIla, recombinant activated factor VII (eptacog alfa); APCC, activated prothrombin complex concentrate; rpFVIII: recombinant porcine factor VIII (susoctocog alfa), hFVIII, human (plasma-derived or recombinant) factor VIII; h: hour; d: day.

bleeds treated with rFVIIa, were analyzed. The reported median initial rFVIIa dose was $90-105 \mu \mathrm{g} / \mathrm{kg}$ body weight, with ranges between $25-181 \mu \mathrm{g} / \mathrm{kg}$ in individual studies. Doses were repeated mostly every $2-3 \mathrm{~h}$, with the median total number of doses being 10-28. rFVIIa was used as first-line treatment in the majority of cases, and 39-90\% of treated bleeds were severe. There was considerable variability across the 12 studies in terms of how hemostatic effectiveness was defined. The only effectiveness outcome that provided sufficient data was defined as 'complete' or 'partial response', available for six studies. In five of these six studies, treatment efficacy was $>90 \%$, at both patient and bleed levels.

The safety of rFVIIa in AHA was also addressed in the same systematic review. ${ }^{37}$ Thromboembolic and cardiovascular events were assessed in eight of the 12 studies and reported in $0-5 \%$ of patients. Mortality was included in ten of the studies, with eight reporting no mortality related to rFVIIa. The two studies that reported deaths from thromboembolic events potentially related to rFVIIa were a Japanese surveillance study (2 deaths in 132 rFVIIatreated patients) ${ }^{39}$ and the GTH study (3 deaths in 61 rFVIIa-treated patients). ${ }^{10}$ One patient in the GTH study died from portal vein thrombosis on day 6 while on rFVIIa for 3 days. The other two patients died of ischemic stroke on days 5 and 35 of rFVIIa treatment; these two patients received $\mathrm{rFVII}$ a together with tranexamic acid. In EACH2, thrombotic events were reported in 5/174 (2.9\%) patients in association with rFVIIa treatment. ${ }^{8}$

For initial treatment with rFVIIa, we recommend bolus injections of $90 \mu \mathrm{g} / \mathrm{kg}$ every 2-3 h until hemostasis is achieved (GRADE 1B).

\section{Activated prothrombin complex concentrate}

APCC is used for the treatment and prevention of bleeds in patients with congenital hemophilia with inhibitors, ${ }^{40-42}$ and is widely used for AHA, although no systematic reviews are available. The recommended dose is $50-100 \mathrm{U} / \mathrm{kg}$ every $8-12 \mathrm{~h}$, up to a maximum of 200 $\mathrm{U} / \mathrm{kg} /$ day. ${ }^{1}$ In the EACH2 registry, propensity scorematched analysis indicated indistinguishable efficacy for APCC compared with rFVIIa, with $>90 \%$ bleed control rates when used as first-line treatment. ${ }^{8}$ An earlier study collected data from 34 cases in three centers in the USA over 10 years. $^{43} \mathrm{~A}$ total of 55 bleeding events were observed and the response rate was $76 \%$ and $100 \%$ for patients with severe and moderate bleeds, respectively. ${ }^{43}$ APCC has also been used for secondary prophylaxis. ${ }^{44,45}$

In a retrospective study, APCC exhibited a favorable safety profile indicating that it is well-tolerated with few adverse events. ${ }^{46}$ Thrombotic events, including myocardial infarction and venous thrombosis, were mostly reported in patients with additional risk factors. ${ }^{46,47}$ In EACH2, thrombotic events with APCC were reported in $3 / 63$ $(4.8 \%)$ patients. ${ }^{8}$ Disseminated intravascular coagulation has been observed following APCC administration in some patients receiving doses higher than $200 \mathrm{U} / \mathrm{kg} /$ day. ${ }^{47}$ APCC is contraindicated in patients with signs of disseminated intravascular coagulation.

For initial treatment with APCC, we recommend bolus injections of between 50-100 U/kg every 8-12 h, up to a maximum of $200 \mathrm{U} / \mathrm{kg} /$ day (GRADE 1B).

\section{Recombinant porcine factor VIII}

Although animal-derived concentrates are no longer available, porcine plasma-derived FVIII concentrate was used extensively in the past for the treatment of AHA because anti-FVIII autoantibodies often exhibited low cross-reactivity with porcine FVIII. ${ }^{48}$ rpFVIII (susoctocog alfa) was assessed in a prospective, single-arm clinical study. ${ }^{15}$ Patients with AHA and a serious bleed were eligible, but were excluded if they had an anti-rpFVIII inhibitor 
titer $>20$ BU. rpFVII was administered at an initial dose of $200 \mathrm{U} / \mathrm{kg}$. Subsequent doses and dosing intervals were assigned by the treating physician based on the clinical response and FVIII:C measured, with the aim of maintaining FVIII:C $>80 \%$ for severe bleeds of particular concern" (e.g. severe mucous, intracranial, retro- or intra-abdominal, genitourinary, neck, traumatic or postoperative bleeds), and $>50 \%$ for all other bleeds. Clinical assessment after $24 \mathrm{~h}$ indicated an effective response in $24 / 28$ patients and a partial response in $4 / 28$ patients. The bleeding was controlled at the time of the final dose in $24 / 28(86 \%)$ patients. FVIII levels measured immediately after the first dose and $24 \mathrm{~h}$ later were $22-540 \%$ and $2-369 \%$ of normal, respectively. The presence of cross-reacting antibodies did not appear to affect the clinical response after $24 \mathrm{~h}$, but patients with cross-reactivity needed higher doses in the first $24 \mathrm{~h}$ than patients without cross-reactivity (median 1,400 and 300 $\mathrm{U} / \mathrm{kg}$, respectively). Cross-reacting anti-rpFVIII inhibitors were found in $44 \%$ of patients in a recent study. ${ }^{49} \mathrm{~A}$ retrospective study used a lower starting dose of $100 \mathrm{U} / \mathrm{kg}$ in six of seven patients given rpFVIII as second-line therapy, with five of these six patients achieving FVIII:C $>100 \%$ after infusion (one patient had unmeasurable FVIII:C). ${ }^{50}$ Overall, treatment was effective in five of the seven patients. These data suggest that, although the approved initial dose for $\mathrm{rpFVIII} \mathrm{is} 200 \mathrm{U} / \mathrm{kg}$, an initial dose of $100 \mathrm{U} / \mathrm{kg}$ appears to be sufficient for most patients, and FVIII:C should be closely monitored to prevent excess FVIII:C. The baseline antirpFVIII titer may help to identify those patients for whom rpFVIII is unlikely to be efficacious.

In the pivotal trial, rpFVIII was well-tolerated in all patients. ${ }^{15}$ A total of $5 / 18$ patients who did not have antirpFVIII inhibitors at baseline developed de novo antirpFVIII antibodies after 8-85 days, prompting discontinuation of the drug in two patients. No patient developed thromboembolic events.

For initial treatment with rpFVIII, we recommend the approved dose of $200 \mathrm{U} / \mathrm{kg}$, followed by further doses to maintain trough levels $>50 \%$ (GRADE 1B).

We recommend close monitoring of FVIII activity during therapy with rpFVIII (GRADE 1B).

\section{Human factor VIII concentrates and desmopressin}

The 2009 international AHA recommendations suggested the use of human FVIII concentrates or desmopressin only if therapy with bypassing agents was not available. ${ }^{1}$ In the EACH2 registry, an efficacy assessment of the treatment of first bleeding episodes could be performed in 288 patients, of whom $219(76 \%)$ received bypassing agents and $69(24 \%)$ received human FVIII or desmopressin. ${ }^{8}$ Patients administered human FVIII concentrates or desmopressin had higher initial FVIII levels, lower inhibitor titers, less severe bleeds and received tranexamic acid more often. When comparing propensity score-matched groups $(n=60$ per group), significantly lower efficacy rates were observed for treatment with FVIII or desmopressin (68\%) compared with bypassing agents (93\%). Of note, there was no matching for tranexamic acid use in this comparison. There may be a risk of fluid overload, heart failure and severe hyponatremia with desmopressin and it should not, therefore, be used in elderly patients. ${ }^{51}$

We suggest the use of recombinant or plasma-derived human FVIII concentrates only if bypassing agents or rpFVIII are unavailable or ineffective and the inhibitor titer is low. We recommend against the use of desmopressin (GRADE 1B).

\section{Anti-fibrinolytics and other treatments}

Some controversy persists regarding the use of anti-fibrinolytic agents in conjunction with bypassing agents. Anti-fibrinolytic agents should be used with caution in patients treated with APCC. ${ }^{52}$ However, in a small case series of combination therapy of tranexamic acid with APCC, which included one patient with AHA, no thromboembolic events or disseminated intravascular coagulation were reported. ${ }^{53}$ Combining tranexamic acid with rFVIIa has been of less concern traditionally. ${ }^{54}$ In the EACH2 registry, $17 \%$ of rFVIIa patients received ancillary anti-fibrinolytic therapy compared with only $5 \%$ of patients treated with APCC. ${ }^{8}$ In the GTH study, 32/102 (31\%) patients received tranexamic acid, 63/102 (62\%) received rFVIIa, and $21(21 \%)$ received both concomitantly. ${ }^{10}$ Of the three fatal thromboembolic events observed in relation to rFVIIa, two occurred in the 21 patients receiving concomitant tranexamic acid and rFVIIa. ${ }^{10} \mathrm{We}$, therefore, suggest caution when combining tranexamic acid with bypassing agents. Topical tranexamic acid may be used as an alternative for some types of bleeding.

A plasma-derived, purified FVIIa/factor X concentrate (MC710, Byclot) is approved in Japan for the treatment of acute bleeds in patients with inhibitors against FVIII or FIX ${ }^{55}$ Studies in AHA are not available.

Emicizumab is a recombinant, humanized, bispecific monoclonal antibody with FVIII-mimetic activity. It was recently licensed for prophylaxis in patients with hemophilia A with or without inhibitors. ${ }^{56}$ The drug has been used in a few patients with AHA with severe bleeding. ${ }^{57,58}$ However, the safety and efficacy of emicizumab in AHA is unknown and so this antibody should not be used in this group of patients outside of clinical trials. This is particularly relevant for elderly patients, in whom cardiovascular events are more common, and in women who are considering use of hormonal therapy or future pregnancy, as emicizumab is an IgG4 antibody and may therefore be transferred across the placenta. Given its long half-life of approximately 1 month, patients treated with emicizum$a b$ are exposed for up to 6 months after their last dose.

\section{Invasive procedures}

In patients with AHA, even minor invasive procedures can result in significant bleeding; ${ }^{36,59}$ therefore, particular caution should be exercised during all procedures and surgery and, if possible, they should be delayed until after the inhibitor has been eradicated. Use of a bypassing agent or rpFVIII is usually required for central venous access, taking biopsies or performing other invasive procedures.

We recommend the prophylactic use of bypassing agents or $r p F V I I I$ to cover minor or major invasive procedures (GRADE 1B).

\section{Immunosuppressive therapy}

\section{Goals of immunosuppressive therapy and definition of remission}

The goal of immunosuppressive therapy (IST) is to reduce the risk of bleeding by shortening the time to achieve remission of AHA. Spontaneous remissions have been observed in patients not treated with IST, but this outcome is unpredictable. ${ }^{60}$ Definitions of remission vary across studies and registries. The UK surveillance study defined complete remission as: FVIII normal, inhibitor undetectable, and immunosuppression stopped or 


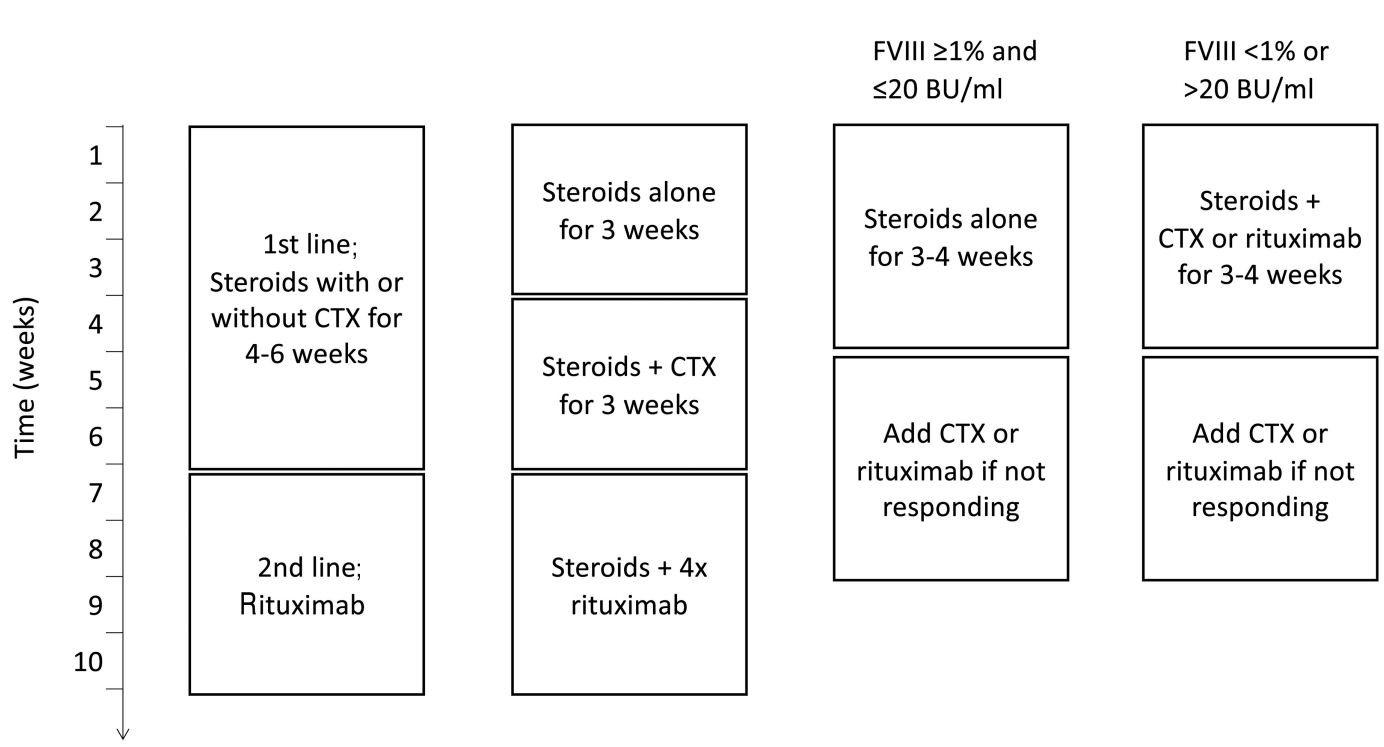

Figure 3. Recommendations regarding Immunosuppressive therapy in patients with acquired hemophilia A. Comparison of immunosuppressive therapy regimens recommended in the 2009 international recommendations by Huth-Kühne et al. ${ }^{1}$ the GTH study ${ }^{10}$ and the current paper. FVIII; factor VIII activity; BU: Bethseda unit; CTX, cyclophosphamide.

reduced to doses used before AHA developed without relapse. ${ }^{2}$ The GTH study also included a definition for partial remission: FVIII restored to $>50 \%$ and no bleeding after stopping any hemostatic treatment for at least $24 \mathrm{~h} \cdot{ }^{10}$

\section{General strategy and prognostic factors}

The 2009 international AHA guidelines recommended starting IST in all patients with AHA immediately following diagnosis. ${ }^{1}$ This recommendation was mainly based on the high bleed-related mortality in earlier studies and the observation that the initial bleeding tendency was not predictive of later major or even fatal bleeding. ${ }^{61}$ Initial treatment with corticosteroids alone or in combination with cyclophosphamide was recommended for up to 6 weeks, while second-line therapy with rituximab was suggested if first-line IST failed or was contraindicated (Figure 3).

The GTH-AH 01/2010 study protocol was designed as a variant of this recommendation and was the first IST protocol investigated prospectively. ${ }^{10}$ Patients were enrolled $\leq 7$ days after starting any IST, and follow-up data were collected weekly until complete remission was achieved. The 1 -year survival rate was $68 \%$. The most frequent cause of death among the 34 patients who died was infection $(\mathrm{n}=16)$, followed by cardiovascular disorders $(n=6)$, underlying disorders $(n=3)$, and bleeding $(n=3)$. Fourteen deaths were considered to be directly related to IST. These results established that IST-related mortality, in particular infection, exceeds the current risk of fatal bleeding in AHA. Patients with a poor World Health Organization (WHO) performance status $(>2)$ at presentation had a 4-fold increased risk of mortality. Therefore, careful individual consideration of the requirement for and contraindications against IST, its intensity and timing is warranted in frail patients with AHA and should be a priority for future research. IST should be stopped if severe side effects to treatment develop.
We recommend IST in all patients with AHA. However, particular caution should be exercised in frail patients (GRADE 1B).

The success of IST, in particular the time to achieve remission, varies largely among patients. In the GTH study, 85/102 (83\%) patients achieved a partial remission after a median time of 5 weeks (range, 1-52 weeks). ${ }^{10}$ FVIII:C at presentation was the most important prognostic factor; patients with FVIII $<1 \%$ achieved partial remission less often compared with other patients (77 vs. 89\%, respectively) and after a significantly longer time on IST (43 vs. 24 days, respectively). Anti-FVIII IgA autoantibodies were predictors of relapse as observed in $45 \%$ of patients with anti-FVIII IgA titers $>1: 80$. These data suggest that IST should be individualized in patients with AHA according to baseline characteristics, although interventional studies with stratified protocols have not been performed.

We suggest using prognostic markers (FVIII activity, inhibitor titer, if available) to individualize IST (GRADE 2B).

The benefit of combining corticosteroids with other immunosuppressants for first-line therapy is uncertain and cannot be concluded from the published observational studies. Combination therapy shortened the time to remission in the EACH2 registry but not in the UK surveillance study. ${ }^{2}$ GTH data indicated that achieving partial remission within 21 days of steroid therapy was unlikely (negative predictive value, $84 \%$ ) if patients had FVIII $<1 \%$ or an inhibitor titer $>20 \mathrm{BU} .{ }^{10} \mathrm{We}$, therefore, suggest combination therapy in these patients. In all other patients (i.e., those with FVIII $\geq 1 \%$ and inhibitor $\leq 20 \mathrm{BU}$ ), we suggest using steroids alone, because the benefit of more intense regimens is uncertain, and the risk of infectious complications currently exceeds the risk of bleeding.

We suggest that patients with FVIII $\geq 1$ IU/dL and inhibitor titer $\leq 20 \mathrm{BU}$ at baseline receive first-line treatment with corticosteroids alone for 3-4 weeks (GRADE 2B).

We suggest combining corticosteroids with rituximab or a cyto- 
toxic agent for first-line therapy in patients with FVIII $<1$ IU/dL or inhibitor titer $>20 \mathrm{BU}$ (GRADE 2B).

In the GTH study, 23 patients had a continuous improvement of their FVIII:C and/or inhibitor titer while not achieving partial remission during the first 3 weeks on steroids. ${ }^{10}$ These patients were not escalated to second-line IST and all achieved partial remission after a median of 29 days.

We suggest extending observation in patients who do not achieve remission with first-line IST but have continued improvement of FVIII activity and inhibitor titer (GRADE 2B).

Patients not responding to steroids after 3 weeks were escalated to second-line therapy with cyclophosphamide, and later rituximab, in the GTH study. Eighty-three percent of these patients achieved remission.

We suggest second-line therapy with rituximab or a cytotoxic agent, whichever was not used during first-line therapy (GRADE 1B).

\section{Corticosteroids}

Corticosteroid therapy with prednisolone or prednisone $1 \mathrm{mg} / \mathrm{kg} /$ day $\mathrm{PO}$ for 4-6 weeks was suggested in the 2009 international AHA recommendations. ${ }^{1}$ This regimen was also used in the GTH study. ${ }^{10}$

For corticosteroid therapy, we suggest prednisolone or prednisone at a dose of $1 \mathrm{mg} / \mathrm{kg} /$ day $P O$ for a maximum of 4-6 weeks (followed by tapered withdrawal) (GRADE 2B).

\section{Rituximab}

Second-line therapy with rituximab for inhibitor eradication was recommended in the 2009 international AHA recommendations. ${ }^{1}$ Rituximab is often used at the licensed dose for the treatment of non-Hodgkin lymphoma (i.e., $375 \mathrm{mg} / \mathrm{m}^{2} /$ week), but has also been used in lower doses of $100 \mathrm{mg} /$ week in some reported cases. ${ }^{62-65}$ The drug is licensed for the treatment of rheumatoid arthritis at a fixed dose of $1,000 \mathrm{mg}$ on days 1 and $15 .^{66}$ This regimen has also been used in immune thrombocytopenia. ${ }^{67,68}$ Although not licensed for the treatment of AHA, we suggest adding rituximab to first-line IST in patients with poor prognostic markers, in those with contraindications against corticosteroids, or as second-line therapy.

We suggest rituximab at a dose of $375 \mathrm{mg} / \mathrm{m}^{2}$ weekly for a maximum of four cycles (GRADE 2B).

\section{Cytotoxic agents}

Cyclophosphamide therapy was recommended at a dose of $1.5-2 \mathrm{mg} / \mathrm{kg} /$ day $\mathrm{PO}$ in the 2009 international AHA recommendations. ${ }^{1}$ Intravenous pulse therapy of cyclophosphamide $500-1,000 \mathrm{mg} / \mathrm{m}^{2}$ is licensed for the treatment of severe lupus nephritis and Wegener granulomatosis, although there is no experience in AHA. ${ }^{69}$ Recently, the use of mycophenolate mofetil (MMF) was reported in a retrospective collection of 11 AHA patients. ${ }^{70} \mathrm{MMF}$ was started at $1 \mathrm{~g} /$ day and increased to 2 g/day after 1 week. Among seven patients given MMF together with prednisolone as first-line IST, one complete and five partial remissions were achieved after 4 to 77 weeks; among four patients receiving MMF for secondline IST after a median of 14 weeks of other treatments, three complete remissions and one parital remission were observed. Close monitoring for leukopenia, thrombocytopenia, and infections is required during treatment with any cytotoxic agent. These agents should not be used in pregnancy or in women who are breastfeeding.
As cytotoxic therapy, we suggest cyclophosphamide at a dose of $1.5-2 \mathrm{mg} / \mathrm{kg} /$ day $P O$ for a maximum of 6 weeks, or MMF at a dose of $1 \mathrm{~g} /$ day for 1 week, followed by $2 \mathrm{~g} /$ day (GRADE 2B).

\section{Immunoadsorption, immune tolerance regimens and immunoglobulins}

Immunoadsorption protocols have been used successfully to deplete inhibitors in patients with AHA. The modified Bonn-Malmö protocol combined immunoadsorption with high-dose FVIII, intravenous immunoglobulins and immunosuppression. ${ }^{71}$ Inhibitors were eliminated after a median of 3 days. Whether the overall response rate is superior to that achieved with IST alone remains unclear, as does the relative contribution of FVIII and immunoglobulins in this regimen. We, therefore, suggest that this protocol is used only in patients with particularly severe bleeding or those who are resistant to other therapy.

Similarly to immune tolerance in hemophilia A with inhibitors, high-dose FVIII has been combined with IST by several investigators in AHA. ${ }^{71-74}$ However, a conclusion as to whether FVIII increased the efficacy of IST cannot be reached based on these studies due to the lack of a control group for comparison. We, therefore, suggest this treatment only in patients with life-threatening bleeding in whom the available hemostatic therapies have failed.

We do not recommend the use of high-dose human FVIII for immune tolerance induction in AHA (GRADE 2C).

High-dose intravenous immunoglobulins are known to be effective in idiopathic thrombocytopenic purpura; however, they had little or no effect in patients with AHA in the one currently available study on this therapeutic strategy. $^{75}$

We do not recommend use of high-dose intravenous immunoglobulins for inhibitor eradication in patients with AHA (GRADE 1B).

\section{Follow-up}

Adverse events occurred in $66 \%$ of patients in the GTH registry (including IST-related events in 50\%): $25 \%$ of the events occurred after more than 100 days. ${ }^{10}$ Relapse occurred in $12-18 \%$ of EACH2 patients after a median time of 138 days. ${ }^{7}$ Given the risks of adverse events and of recurrence, patients should be closely monitored until they achieve complete remission and for several months thereafter. Monitoring of FVIII:C is more sensitive than APTT for detecting recurrence.

We recommend follow-up after complete remission, using FVIII:C monitoring monthly during the first 6 months, every 2-3 months up to 12 months, and every 6 months during the second year and beyond, if possible (GRADE 1B).

\section{Pregnancy-associated acquired hemophilia A}

Hemostatic therapy and the response to it were similar in 42 women with pregnancy-associated AHA as compared with other patients in the EACH2 registry. ${ }^{5} \mathrm{~A}$ total of $70 \%$ of the women received steroids alone for IST, while the rest received steroids and rituximab, cytotoxic agents, or intravenous immunoglobulins. The success rates and times to remission were similar in these women compared with those in other AHA patients in the registry. The mortality rate was lower than in the other patients, possibly because of the younger age of women with pregnancy-associated AHA, and the risk of relapse 
appeared to be low (2 patients). We do not, therefore, recommend a different approach for IST in pregnancy-associated AHA, except for more careful consideration regarding the use of cytotoxic agents in women of childbearing age because of the potential of these drugs to reduce fertility and cause embryotoxicity.

In women with pregnancy-associated AHA, we suggest the same approach for IST as in other patients, but with more careful consideration regarding the use of cytotoxic agents (GRADE 2C).

\section{Thromboprophylaxis}

Cardiovascular events, including thrombosis, myocardial infarction and stroke, were recorded as the cause of death in $6-7 \%$ of patients with AHA in the GTH and SACHA registries. ${ }^{9,10}$ It therefore appears justified to recommend thromboprophylaxis according to the 2018 American
Society of Hematology (ASH) guidelines in non-bleeding patients whose FVIII:C has returned to normal. ${ }^{76}$ If an indication for antiplatelet drugs (e.g., history of myocardial infarction or stroke) or oral anticoagulants (e.g., atrial fibrillation, artificial heart valves or recurrent venous thromboembolism) exists, the use of these drugs should be initiated after FVIII has returned to normal levels.

We recommend thromboprophylaxis according to ASH guidelines if FVIII:C has returned to normal levels. If indicated, therapy with anti-platelet drugs or oral anticoagulants should be initiated, after normal FVIII:C levels have been achieved (GRADE 1C).

\section{Acknowledgments}

Editorial support was provided by Physicians World GmbH Mannheim, Germany, supported by Novo Nordisk Health Care $A G$, Zürich, Switzerland.

\section{References}

1. Huth-Kuhne A, Baudo F, Collins $\mathrm{P}$, et al. International recommendations on the diagnosis and treatment of patients with acquired hemophilia A. Haematologica. 2009;94(4):566-575

2. Collins PW, Hirsch S, Baglin TP, et al. Acquired hemophilia A in the United Kingdom: a 2-year national surveillance study by the United Kingdom Haemophilia Centre Doctors' Organisation. Blood. 2007;109(5):1870-1877

3. Knoebl P, Marco P, Baudo F, et al. Demographic and clinical data in acquired hemophilia A: results from the European Acquired Haemophilia Registry (EACH2). J Thromb Haemost. 2012;10(4):622-631.

4. Napolitano M, Siragusa S, Mancuso S, Kessler CM. Acquired haemophilia in cancer: a systematic and critical literature review. Haemophilia. 2018;24(1):43-56.

5. Tengborn L, Baudo F, Huth-Kuhne A, et al. Pregnancy-associated acquired haemophilia A: results from the European Acquired Haemophilia (EACH2) registry. BJOG. 2012;119(12):1529-1537.

6. Collins $\mathrm{P}$, Chalmers E, Hart D, et al. Diagnosis and management of acquired coagulation inhibitors: a guideline from UKHCDO. Br J Haematol. 2013;162(6):758773.

7. Collins P, Baudo F, Knoebl P, et al. Immunosuppression for acquired hemophilia A: results from the European Acquired Haemophilia Registry (EACH2). Blood. 2012;120(1):47-55

8. Baudo F, Collins P, Huth-Kuhne A, et al. Management of bleeding in acquired hemophilia A: results from the European Acquired Haemophilia (EACH2) Registry. Blood. 2012;120(1):39-46.

9. Borg JY, Guillet B, Le Cam-Duchez V, Goudemand J, Levesque H, Group SS. Outcome of acquired haemophilia in France: the prospective SACHA (Surveillance des Auto antiCorps au cours de l'Hemophilie Acquise) registry. Haemophilia. 2013;19(4): 564-570.

10. Tiede A, Klamroth R, Scharf RE, et al. Prognostic factors for remission of and survival in acquired hemophilia A (AHA): results from the GTH-AH 01/2010 study. Blood. 2015;125(7):1091-1097.
11. Tiede A, Hofbauer CJ, Werwitzke S, et al. Anti-factor VIII IgA as a potential marker of poor prognosis in acquired hemophilia $\mathrm{A}$ : results from the GTH-AH 01/2010 study. Blood. 2016;127(19):2289-2297.

12. Holstein K, Liu X, Smith A, et al. Factor VIII activity and risk of bleeding in acquired hemophilia A: results from the GTH-AH 01/2010 study. Blood. 2020 Feb 20. [Epub ahead of print]

13. Werwitzke S, Geisen U, Nowak-Gottl U, et al. Diagnostic and prognostic value of factor VIII binding antibodies in acquired hemophilia A: data from the GTH-AH 01/2010 study. J Thromb Haemost. 2016;14(5):940947.

14. Ma AD, Kessler CM, Al-Mondhiry HA, Gut RZ, Cooper DL. Use of recombinant activated factor VII for acute bleeding episodes in acquired hemophilia: final analysis from the Hemostasis and Thrombosis Research Society Registry acquired hemophilia study. Blood Coagul Fibrinolysis. 2016;27(7):753760.

15. Kruse-Jarres R, St-Louis J, Greist A, et al. Efficacy and safety of OBI-1, an antihaemophilic factor VIII (recombinant), porcine sequence, in subjects with acquired haemophilia A. Haemophilia. 2015;21(2): 162-170.

16. Guyatt G, Oxman AD, Akl EA, et al. GRADE guidelines: 1 . Introduction-GRADE evidence profiles and summary of findings tables. J Clin Epidemiol. 2011;64(4):383-394

17. Chee YL, Crawford JC, Watson HG Greaves M. Guidelines on the assessment of bleeding risk prior to surgery or invasive procedures. Br J Haematol. 2008;140(5):496 504.

18. Lossing TS, Kasper CK, Feinstein DI Detection of factor VIII inhibitors with the partial thromboplastin time. Blood. 1977;49(5):793-797.

19. Tiede A, Werwitzke S, Scharf RE. Laboratory diagnosis of acquired hemophilia A: limitations, consequences, and challenges. Semin Thromb Hemost. 2014;40(7): 803-811.

20. Kasper CK. Laboratory tests for factor VIII inhibitors, their variation, significance and interpretation. Blood Coagul Fibrinolysis. 1991;2(Suppl 1):7-10.

21. Blanco AN, Cardozo MA, Candela M Santarelli MT, Perez Bianco R, Lazzari MA. Anti-factor VIII inhibitors and lupus antico- agulants in haemophilia A patients. Thromb Haemost. 1997;77(4):656-659.

22. Chandler WL, Ferrell C, Lee J, Tun T, Kha H. Comparison of three methods for measuring factor VIII levels in plasma. Am J Clin Pathol. 2003;120(1):34-39.

23. de Maistre E, Wahl D, Perret-Guillaume C, et al. A chromogenic assay allows reliable measurement of factor VIII levels in the presence of strong lupus anticoagulants. Thromb Haemost. 1998;79(1):237-238.

24. Taher A, Abiad R, Uthman I. Coexistence of lupus anticoagulant and acquired haemophilia in a patient with monoclonal gammopathy of unknown significance. Lupus. 2003;12(11):854-856.

25. Seethala S, Collins NP Jr, Comerci G Jr. An unusual etiology for elevation of activated partial thromboplastin time (aPTT) in SLE: acquired hemophilia and lupus anticoagulant. Case Report Hematol. 2013;2013: 521785.

26. Kasper CK, Aledort L, Aronson D, et al. Proceedings: a more uniform measurement of factor VIII inhibitors. Thromb Diath Haemorrh. 1975;34(2):612.

27. Gawryl MS, Hoyer LW. Inactivation of factor VIII coagulant activity by two different types of human antibodies. Blood. 1982;60(5):1103-1109.

28. Kitchen S, McCraw A, Echenagucia M Diagnosis of Haemophilia and Other Bleeding Disorders (second edition). World Federation of Hemophilia, 2010.

29. Rampersad AG, Boylan B, Miller $\mathrm{CH}$ Shapiro A. Distinguishing lupus anticoagulants from factor VIII inhibitors in haemophilic and non-haemophilic patients. Haemophilia. 2018;24(5):807-814.

30. Verbruggen B, Novakova I, Wessels H, Boezeman J, van den Berg M, MauserBunschoten E. The Nijmegen modification of the Bethesda assay for factor VIII:C inhibitors: improved specificity and reliability. Thromb Haemost. 1995;73(2):247-251.

31. Batty P, Platton S, Bowles L, Pasi KJ, Hart DP Pre-analytical heat treatment and a FVIII ELISA improve factor VIII antibody detection in acquired haemophilia A. Br J Haematol. 2014;166(6):953-956.

32. Boylan B, Miller CH. Effects of pre-analyti$\mathrm{cal}$ heat treatment in factor VIII (FVIII) inhibitor assays on FVIII antibody levels. Haemophilia. 2018;24(3):487-491.

33. Pham TV, Sorenson CA, Nable JV. Acquired 
factor VIII deficiency presenting with compartment syndrome. Am J Emerg Med. 2014;32(2):195.

34. Dachman AF, Margolis H, Aboulafia E. Does Sjogren's syndrome predispose surgical patients to acquired hemophilia? J Am Osteopath Assoc. 1995;95(2):115-118, 121.

35. Lusher JM. Early treatment with recombinant factor VIIa results in greater efficacy with less product. Eur J Haematol Suppl. 1998;63:7-10

36. Delgado J, Jimenez-Yuste V, HernandezNavarro F, Villar A. Acquired haemophilia: review and meta-analysis focused on therapy and prognostic factors. Br J Haematol. 2003;121(1):21-35

37. Tiede A, Worster A. Lessons from a systematic literature review of the effectiveness of recombinant factor VIIa in acquired haemophilia. Ann Hematol. 2018;97(10): 1889-1901.

38. Tiede A, Giangrande P, Teitel J, et al. Clinical evaluation of bleeds and response to haemostatic treatment in patients with acquired haemophilia: a global expert consensus statement. Haemophilia. 2019;25(6):969978.

39. Seita I, Amano K, Higasa S, Sawada A, Kuwahara M, Shima M. Treatment of acute bleeding episodes in acquired haemophilia with recombinant activated factor VII (rFVIIa): analysis from 10-year Japanese postmarketing surveillance. J Thromb Haemost. 2013;11(Suppl. 2):119.

40. Dimichele D, Negrier C. A retrospective postlicensure survey of FEIBA efficacy and safety. Haemophilia. 2006;12(4):352-362

41. Leissinger CA, Becton DL, Ewing NP, Valentino LA. Prophylactic treatment with activated prothrombin complex concentrate (FEIBA) reduces the frequency of bleeding episodes in paediatric patients with haemophilia A and inhibitors. Haemophilia. 2007;13(3):249-255

42. Tjonnfiord GE, Holme PA. Factor eight inhibitor bypass activity (FEIBA) in the management of bleeds in hemophilia patients with high-titer inhibitors. Vasc Health Risk Manag. 2007;3(4):527-531

43. Sallah S. Treatment of acquired haemophilia with factor eight inhibitor bypassing activity. Haemophilia. 2004;10(2):169-173,

44. Zanon E, Milan M, Gamba G, et al. Activated prothrombin complex concentrate (FEIBA®) for the treatment and prevention of bleeding in patients with acquired haemophilia: a sequential study. Thromb Res. 2015;136(6):1299-1302.

45. Arokszallasi A, Razso K, Ilonczai P, et al. A decade-long clinical experience on the prophylactic use of activated prothrombin complex concentrate in acquired haemophilia A: a case series from a tertiary care centre. Blood Coagul Fibrinolysis. 2018;29(3):282287

46. Aledort LM. Comparative thrombotic event incidence after infusion of recombinant factor VIIa versus factor VIII inhibitor bypass activity. J Thromb Haemost. 2004;2(10): 1700-1708.

47. Ehrlich HJ, Henzl MJ, Gomperts ED. Safety of factor VIII inhibitor bypass activity (FEIBA): 10-year compilation of thrombotic adverse events. Haemophilia. 2002;8(2):8390

48. Giangrande PL. Porcine factor VIII Haemophilia. 2012;18(3):305-309

49. Turkantoz H, Konigs C, Knobl P, et al. Crossreacting inhibitors against recombinant porcine factor VIII in acquired hemophilia A: data from the GTH-AH 01/2010 study. J Thromb Haemost. 2020;18(1):36-43.

50. Tarantino MD, Cuker A, Hardesty B, Roberts JC, Sholzberg M. Recombinan porcine sequence factor VIII (rpFVIII) for acquired haemophilia A: practical clinical experience of its use in seven patients. Haemophilia. 2017;23(1):25-32.

51. EMC. Summary of Product Characteristics DDAVP/Desmopressin Injection 2019 Available from: https://www.medicines. org.uk/emc/product/5447/smpc [Accessed 13 Apr 2020].

52. Baxter Corp. FEIBA VH, Anti-inhibitor coagulant complex, Vapor-Heated. Summary of Product Characteristics 2005; Available from: https://www.rxlist.com/feiba-vhdrug.htm [Accessed 13 Apr 2020]

53. Holmstrom M, Tran HT, Holme PA.

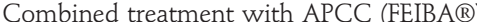
and tranexamic acid in patients with haemophilia A with inhibitors and in patients with acquired haemophilia A--a two-centre experience. Haemophilia. 2012;18(4):544-549.

54. Baudo F, de Cataldo F. Acquired hemophilia: a critical bleeding syndrome. Haematologica. 2004;89(1):96-100.

55. Pharmaceuticals and Medical Devices Agency Japan. Annual Report FY 2014. Available from: http://www.pmda.go.jp/ files/000208305.pdf [Accessed 13 Apr 2020]

56. EMA. Summary of Product Characteristics Hemlibra 2018; Available at https://www.ema.europa.eu/en/documents/product-information/hemlibra-eparproduct-information_en.pdf ; Accessed 13 Apr 2020.

57. Knoebl P, Sperr WR, Schellongowski P, et al. Emicizumab for the treatment of acquired hemophilia A: lessons learned from 4 very different cases. Blood. 2018;132(Suppl 1):2476.

58. Mohnle P, Pekrul I, Spannagl M, Sturm A, Singh D, Dechant C. Emicizumab in the treatment of acquired haemophilia: a case report. Transfus Med Hemother. 2019;46(2): 121-123.

59. Hay CR. Acquired haemophilia. Baillieres Clin Haematol. 1998;11(2):287-303.

60. Lottenberg R, Kentro TB, Kitchens CS. Acquired hemophilia. A natural history study of 16 patients with factor VIII inhibitors receiving little or no therapy. Arch Intern Med. 1987;147(6):1077-1081

61. Hay CR, Brown S, Collins PW, Keeling DM, Liesner $\mathrm{R}$. The diagnosis and management of factor VIII and IX inhibitors: a guideline from the United Kingdom Haemophilia Centre Doctors Organisation. $\mathrm{Br} J$ Haematol. 2006;133(6):591-605

62. Mumoli N, Giorgi-Pierfranceschi M, Ferretti A, Vitale J, Cei M. Acquired hemophilia treated using low-dose of rituximab. J Am Geriatr Soc. 2016;64(8):1744-1745.

63. Wermke $M$, von Bonin $M$, Gehrisch $S$,
Siegert G, Ehninger G, Platzbecker U Successful eradication of acquired factorVIII-inhibitor using single low-dose rituximab. Haematologica. 2010;95(3):521-522.

64. Xu Y, Zhang X, Zhao Y, Zhao L, Oiu H, Wu D. Successful treatment of a patient with acquired haemophilia A with a combination of a low-dose rituximab and recombinant human FVIIa. Haemophilia. 2013;19(2):e9596.

65. Yao Q, Zhu X, Liu Y, et al. Low-dose rituximab in the treatment of acquired haemophilia. Hematology. 2014;19(8):483486.

66. EMA. Summary of Product Characteristics MabThera 2018. Available at: https://www.ema.europa.eu/documents/pr oduct-information/mabthera-epar-productinformation_en.pdf [Accessed 13 Apr 2020].

67. Tran H, Brighton T, Grigg A, et al. A multicentre, single-arm, open-label study evaluating the safety and efficacy of fixed dose rituximab in patients with refractory, relapsed or chronic idiopathic thrombocytopenic purpura (R-ITP1000 study). Br J Haematol. 2014;167(2):243-251.

68. Gudbrandsdottir S, Birgens HS, Frederiksen $\mathrm{H}$, et al. Rituximab and dexamethasone vs dexamethasone monotherapy in newly diagnosed patients with primary immune thrombocytopenia. Blood. 2013;121(11): 1976-1981

69. EMA. Summary of Product Characteristics Cyclophosphamide $500 \mathrm{mg}$ Powder for Solution for Injection or Infusion 2017 Available at: https://www.medicines. org.uk/emc/product $/ 3526 / \mathrm{smpc}$ [Accessed 13 Apr 2020].

70. Obaji S, Rayment R, Collins PW Mycophenolate mofetil as adjunctive therapy in acquired haemophilia A. Haemophilia. 2019;25(1):e59-e65.

71. Zeitler H, Ulrich-Merzenich G, Hess L, et al Treatment of acquired hemophilia by the Bonn-Malmo protocol: documentation of an in vivo immunomodulating concept. Blood. 2005;105(6):2287-2293.

72. Nemes L, Pitlik E. New protocol for immune tolerance induction in acquired hemophilia. Haematologica. 2000;85(10 Suppl):64-68.

73. Lian EC, Villar MJ, Noy LI, Ruiz-Dayao Z Acquired factor VIII inhibitor treated with cyclophosphamide, vincristine, and prednisone. Am J Hematol. 2002;69(4):294295.

74. Huth-Kuhne A, Lages P, Hampel H, Zimmermann R. Management of severe hemorrhage and inhibitor elimination in acquired hemophilia: the modified Heidelberg-Malmö protocol. Haematologica. 2003;88(S12):86-92

75. Schwartz RS, Gabriel DA, Aledort LM, Green D, Kessler CM. A prospective study of treatment of acquired (autoimmune) factor VIII inhibitors with high-dose intravenous gammaglobulin. Blood. 1995;86(2): 797-804.

76. Schunemann HJ, Cushman M, Burnett $A E$ et al. American Society of Hematology 2018 guidelines for management of venous thromboembolism: prophylaxis for hospitalized and nonhospitalized medical patients. Blood Adv. 2018;2(22):3198-3225. 